\title{
FACTORES ASOCIADOS AL DESEMPEÑO DE LA CADENA DE SUMINISTRO AGROALIMENTARIA DE MÉXICO: EL CASO DE LA ZARZAMORA
}

\section{PERFORMANCE RELATED FACTORS IN THE AGRO ALIMENTARY SUPPLY CHAIN IN MEXICO: THE BLACKBERRY CASE}

\author{
Ariel Gutiérrez Ortiz*, Robert Efraín Zárate Cornejo**, \\ Zoe Tamar Infante Jiménez***
}

\begin{abstract}
*Doctor en Ciencias Administrativas y Negocios Internacionales en Facultad de Comercio Exterior de la Universidad de Colima. ORCID: https://orcid.org/0000-0003-1468-0783

**Doctor en Ciencias Económicas en Facultad de Contaduría y Administración de la Universidad Autónoma de Baja California. Email: robertzarate.uabc@gmail.com. ORCID: https://orcid.org/0000-0002-6636-1939 ${ }^{* * *}$ Doctor en Ciencias del Desarrollo Regional en Facultad de Contaduría y Ciencias Administrativas de la Universidad Michoacana de San Nicolás de Hidalgo. Email: zoeinfante@hotmail.com. ORCID: https://orcid.org/0000-0003-0223-2422
\end{abstract}

Dirección para recibir correspondencia: agutierrez18@ucol.mx 
FACTORES ASOCIADOS AL DESEMPEÑO DE LA CADENA DE SUMINISTRO AGROALIMENTARIA DE MÉXICO: EL CASO DE LA ZARZAMORA

RESUMEN

OBJETIVO: Identificar y correlacionar los factores que están asociadas al desempeño de una cadena de suministro agroalimentaria de México, tomando como caso el cultivo de la zarzamora en fresco producida en el valle de Los Reyes, Michoacán

MATERIAL Y MÉTODO: La investigación es de tipo descriptivo-correlacional. Se utilizó el coeficiente de correlación de Spearman y el estadístico de asociación gamma de Goodman y Kruskal para conocer la correlación y asociación, respectivamente, entre las variables independientes y la variable dependiente.

RESULTADOS: Los factores asociados al desempeño de la cadena de suministro agroalimentaria fueron, en orden de importancia: eficiencia/costo, capacidad de respuesta al cliente, flexibilidad, calidad en el producto, calidad en el proceso y las relaciones clienteproveedor.

CONCLUSIONES: Derivado del análisis de los resultados, se logró identificar que todas las variables independientes tienen correlación y cierto grado de asociación con la variable dependiente. Se deben diseñar métricas e indicadores para medir el desempeño de una cadena de suministro, lo que les permite un control adecuado sobre las variables o factores que mayormente influyen en dicho desempeño. Existe interés de los productores sobre el tema de la cadena de suministro, lo cual revela que tienen un compromiso con su empresa.

PALABRAS CLAVE: Cadena de suministro. Desempeño. Agronegocios. Cultivo de zarzamora.

\begin{abstract}
OBJECTIVE: To identify and correlate performance related factors in the agro alimentary supply chain in Mexico, considering the blackberry case produced in Valle de Los Reyes, Michoacan.

MATERIAL AND METHOD: The following is a descriptive correlational study. The Spearman correlation coefficient was used as well as the Goodman and Kruskal gamma association in order to find out the correlation and association respectively among the dependent and independent variables.
\end{abstract}


FACTORES ASOCIADOS AL DESEMPEÑO DE LA CADENA DE SUMINISTRO AGROALIMENTARIA DE MÉXICO: EL CASO DE LA ZARZAMORA

RESULTS: The performance related factors in the agro alimentary supply chain are mentioned in order of importance: efficiency/cost, client capacity response, flexibility, product quality, quality in the process and client-provider relations.

CONCLUSION: It was possible to identify, derived from the results that all the independent variables are correlated and there is certain degree of association with the dependent variable. Metrics and indicators must be designed in order to measure performance in the supply chain, which in result allows control on both the factors and the variables that mostly influence such performance. There is interest on the supply chain theme by producers which in turn indicate commitment towards their company.

KEY WORDS: Supply chain. Performance. Agro business. Blackberry crop.

\section{INTRODUCCIÓN}

La competencia global ha impuesto una enorme presión sobre los proveedores de productos y servicios para transformar y mejorar sus prácticas y operaciones. Las compañías están respondiendo a esta presión con reingeniería y aprovechando de mejor manera sus operaciones con la finalidad de servir de mejor manera a sus clientes. De manera más específica, las firmas están involucradas en el mejoramiento del desempeño de sus cadenas de suministro a través de diversas herramientas estratégicas y operacionales (Talluri y Baker, 2002). Es de suma relevancia conocer el desempeño y eficiencia de las empresas de manera individual; sin embargo, en años recientes el rol está cambiando hacía el conocimiento del desempeño de las cadenas de suministro (Lambert y Cooper, 2000). Esto lleva a la competencia entre redes y cadenas de suministro más que una competencia entre firmas individuales (Christopher, 1998, p. 167).

En la actualidad, el ambiente competitivo de los negocios requiere conocer el desempeño de la cadena de suministro, por lo que en diversos sectores económicos esto se ha convertido en un asunto crítico (Balfaqih, et al., 2016). El trabajo más importante en la cadena de suministro es controlar los flujos físicos del producto. Con la constante variación en la cantidad demandada por el cliente, las empresas tienen que ser más receptivas para satisfacer las necesidades de manera eficiente (Hidayat y Astrellita, 2012). En las últimas dos décadas, la medición del desempeño de una cadena de suministro ha tomado ampliar relevancia e interés para las empresas y expertos 
FACTORES ASOCIADOS AL DESEMPEÑO DE LA CADENA DE SUMINISTRO AGROALIMENTARIA DE MÉXICO: EL CASO DE LA ZARZAMORA

en el área (Agami et al., 2012), por lo que es indispensable conocer los factores que se relacionan al desempeño de la cadena de suministro.

En relación a la aportación práctica, este trabajo identifica las variables que se asocian al desempeño de la cadena de suministro de la zarzamora en fresco, producida en el valle de Los Reyes, Michoacán. Con respecto a la aportación teórica-metodológica, ésta investigación aportará información útil para que los actores involucrados en la cadena de suministro agroalimentaria puedan medir el desempeño individual y colectivo. A pesar de que el sector agroalimentario es de suma importancia para nuestro país y también para el Estado de Michoacán, muy pocas investigaciones sobre este fruto han sido realizadas; es por ello, que con este trabajo de investigación se pretende aportar elementos teóricos y metodológicos para el estudio y análisis de este cultivo desde un punto de vista de los negocios. Cabe destacar, que hasta el momento sólo se plantea la correlación y asociación que existe entre las variables independientes con respecto a la dependiente.

El propósito de esta investigación es identificar y correlacionar las variables que están asociadas al desempeño de una cadena de suministro agroalimentaria de México, tomando como caso de estudio el cultivo de la zarzamora que se produce en el Valle de Los Reyes, Michoacán.

\section{Revisión de literatura}

La cadena de suministro se puede definir como una red de organizaciones independientes y conectadas con trabajo mutuo y de cooperación entre ellas, con la finalidad de controlar, manejar y mejorar el flujo de materiales e información desde el proveedor hasta el consumidor (Christopher, 1998, p. 3). También, puede definirse como una red de actividades físicas y toma de decisiones conectadas por flujos de material e información que cruzan las fronteras organizacionales (Van der Vorst, 2005, p. 14). De acuerdo con Lambert y Cooper (2000) existen cuatro características principales de una cadena de suministro: primero pasa a través de varias etapas de aumento dentro de la organización y entre las organizaciones, coordinación vertical; segundo, incluye muchas empresas independientes, lo que sugiere que la relación gerencial es esencial; tercero, una cadena de suministro incluye un flujo bidireccional entre productos e información y las actividades gerenciales y operacionales; cuarto, los miembros de la cadena tienen por como objetivo cumplir las metas para proveer un alto valor al cliente con un uso óptimo de los recursos. A partir de esta concepción, la cadena de suministro se convierte en un elemento clave que permite replantear los procesos productivos deslocalizados de empresas con altas 
FACTORES ASOCIADOS AL DESEMPEÑO DE LA CADENA DE SUMINISTRO AGROALIMENTARIA DE MÉXICO: EL CASO DE LA ZARZAMORA

exigencias de mercados nacionales e internacionales, que buscan dar una respuesta eficiente al consumidor por medio de la distribución física (Bowersox y Closs, 1996).

La gestión de la cadena de suministro es la coordinación sistémica y estratégica de las funciones tradicionales del negocio y sus tácticas a través de estas funciones dentro de una organización en particular y a través de las empresas dentro de la cadena de suministro con el propósito de mejorar el rendimiento a largo plazo de las organizaciones individuales y la cadena de suministro como un todo (CLM, 2000, p. 5).

La cadena de suministro de los productos perecederos se conforma por un proceso sistémico, en el que los actores interactúan desde el sector primario hasta el consumidor final: proveedores de insumos, productores primarios, distribuidores y comercializadores (ver figura 1), (King y Venturini, 2005). Las empresas líderes forman parte de un sistema integrado con la producción primaria por medio de una buena administración de proveedores externos; apoyados con tecnologías especializadas en la producción e integradas fuertemente con el proceso de distribución y mercadeo especializado en los grandes centros de autoservicio del país (Castañeda et al., 2009).

El producto se mueve normalmente cadena abajo, pero puede haber la necesidad de moverlo cadena arriba cuando hay algún tipo de contaminación o riesgo. Asimismo, los flujos de información viajan cadena abajo (calidad, origen, procesos) y cadena arriba (demanda, precios). Otras áreas de soporte de la cadena incluyen las instituciones que regulan las actividades y productos, y finalmente la infraestructura (telecomunicaciones, puertos) que permiten el movimiento y funcionamiento de la cadena (King y Venturini, 2005).

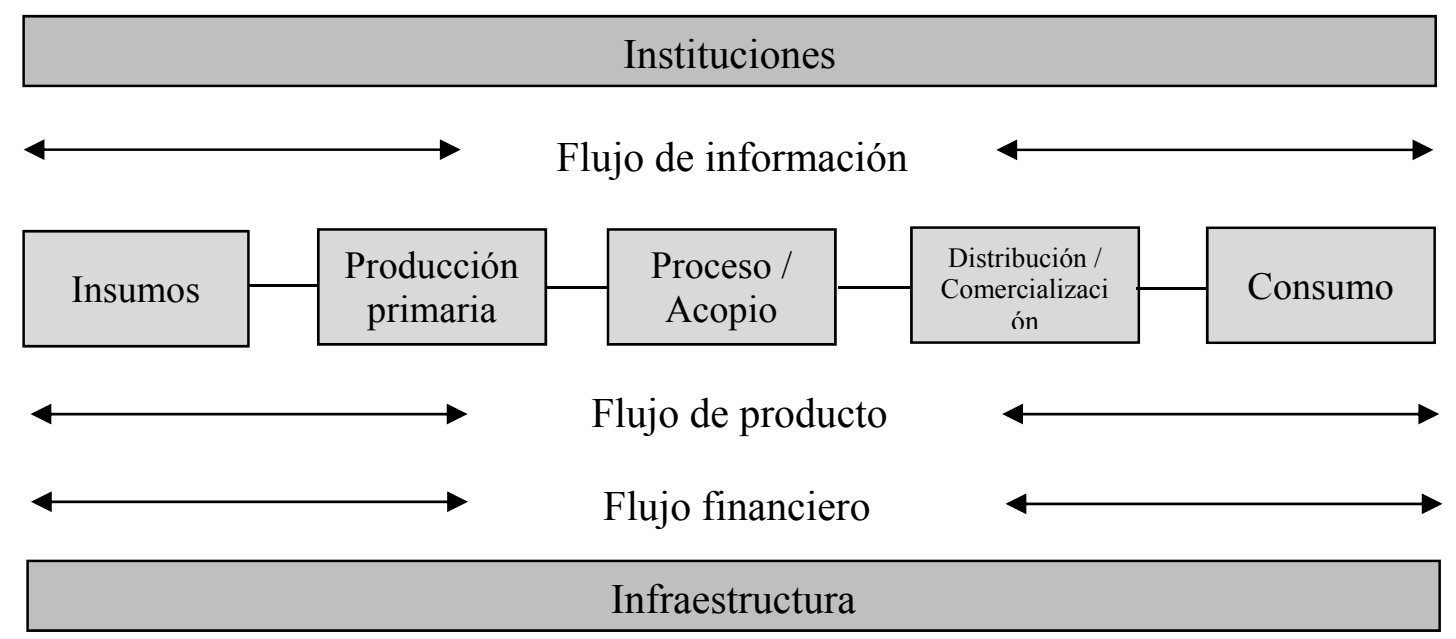

Figura 1. Elementos clave de una cadena de suministro de alimentos de alto valor. Fuente: King y Venturini, 2005. 
FACTORES ASOCIADOS AL DESEMPEÑO DE LA CADENA DE SUMINISTRO AGROALIMENTARIA DE MÉXICO: EL CASO DE LA ZARZAMORA

De acuerdo con Theodoras et al. (2005), existe una amplia brecha de conocimiento entre los agricultores y los procesadores sobre algunos temas; por ejemplo, prácticas de negocios, suministro de productos, expectativas de calidad. Por lo tanto, agricultores y procesadores plantean diferentes preguntas para mejorar el desempeño de la cadena de suministro, que los lleva a correr el riesgo de no especificar de buena manera el proceso de decisión en cada participante de la cadena (Le Heron, 2001).

Debido a la globalización, existe un enorme incremento en los flujos transfronterizos de productos alimenticios, lo que significa que las cadenas de suministro agroalimentarias estén ganando relaciones más complejas. Esto implica el desarrollo e implementación de la mejora de calidad, logística y sistemas de información que permitan que los procesos sean más eficientes y haya un frecuente intercambio masivo de información para lograr una mejor coordinación en los propósitos (Van der Vorst, 2005, p. 14).

La medición del desempeño de la cadena de suministro ofrece a los tomadores de decisiones dentro (productores, distribuidores, vendedores) y fuera (hacedores de política, inversores) información sobre la cadena para la toma de decisiones, desarrollo de políticas, etc. (Gunasekaran et al., 2001). El desempeño de una cadena de suministro puede ser definido por la rentabilidad de la cadena, la cual sólo tiene una fuente de ingresos: el cliente (Chopra y Meindl, 2001). De acuerdo con Van der Vorst (2005, p. 18), el desempeño de la cadena de suministro es el grado en que cumple con los requerimientos del usuario final relacionados con los indicadores de desempeño en cualquier punto del tiempo y a un bajo costo en toda la cadena de suministro. De acuerdo con Neely et al. (2005), la medición del desempeño es el proceso de cuantificar la eficiencia y efectividad de una acción, un indicador de desempeño es una medición utilizada para cuantificar la eficiencia y efectividad de una acción.

En las próximas décadas uno de los desafíos más importantes para los sistemas agroalimentarios del mundo, especialmente para los países en desarrollo, será el asegurar el abasto suficiente de alimentos para su población. Lo anterior, se debe a que la demanda de alimentos se intensificará por el crecimiento de la población, la mayor esperanza de vida; los cambios en los patrones de consumo hacia alimentos más saludables, inocuos, de mejor calidad, producidos de forma amigable con el medio ambiente, cuyo origen pueda ser rastreado por cuestiones de salud y que contengan información especializada en su etiquetado; y, por la demanda de una población madura creciente y con un ingreso disponible mayor (SAGARPA, 2010; FAO, 2009). 
FACTORES ASOCIADOS AL DESEMPEÑO DE LA CADENA DE SUMINISTRO AGROALIMENTARIA DE MÉXICO: EL CASO DE LA ZARZAMORA

La cadena de suministro del sector alimentario mexicano presenta diversas restricciones que la hacen poco competitiva e ineficiente, la heterogeneidad en los canales de distribución, la reducida infraestructura de almacenamiento y transporte, poco uso de estándares de calidad, trazabilidad y normas, las fallas de mercado y de información, los altos costos de transacción, las elevadas mermas y el poco énfasis de la política pública para atender estos problemas son solo algunos de los problemas en los que la política agroalimentaria del país deberá poner especial énfasis (SAGARPA, 2010). Debido a esos múltiples problemas en la medición del desempeño de las cadenas de suministro agroalimentarias, y los diversos cambios profundos que han tenido estas cadenas en los últimos años (por ejemplo, asuntos de seguridad alimentaria, regulaciones al medio ambiente, globalización de los mercados), queda claro que existe una necesidad de contar con más investigaciones en esta área.

A partir de 2005 al año actual 2018, el cultivo de la zarzamora es de relevante importancia en nuestro país y específicamente para el Estado de Michoacán, el cual es el mayor productor a nivel nacional, participando con un 95\% del total de la producción en el país, a nivel estatal se ha ubicado en el tercer lugar como un cultivo de importancia en lo que a valor de la producción se refiere, sólo por detrás del aguacate y del maíz.

El Estado de Michoacán es referente a nivel nacional en la producción de zarzamora en fresco, para el año 2016 representó el 94\% de superficie cosechada a nivel nacional, lo que significó el $94.5 \%$ de la producción en el país. Asimismo, este cultivo representó el tercer lugar en relación al valor de la producción, sólo por debajo del aguacate y el maíz, primero y segundo lugar, respectivamente, para el mismo año. La región de Los Reyes, compuesta por los municipios de Peribán, Los Reyes y Tocumbo, ubicados en el Estado de Michoacán, es la zona de mayor producción de la zarzamora en fresco a nivel nacional y estatal. En el año 2016, dicha región tuvo una producción de más del $60 \%$ con respecto a todo el Estado. La tendencia es a la alza, ya que año con año se suman más productores a la siembra, cosecha y comercialización del cultivo de la zarzamora, incluso productores de otros cultivos transitan hacía este cultivo debido a su alta rentabilidad (SIAP, 2017).

Los problemas y los retos que el sector agroalimentario tiene en materia de la cadena de suministro son diversos, específicamente el cultivo de la zarzamora cuenta con grandes problemas, uno de ellos es sin duda la poca duración o la corta vida de anaquel (perecibilidad) que tiene, disponibilidad inmediata del producto, seguridad alimentaria, frescura, daños en la mercancía debido a la escaza infraestructura carretera para su transporte hacia las empresas 
FACTORES ASOCIADOS AL DESEMPEÑO DE LA CADENA DE SUMINISTRO AGROALIMENTARIA DE MÉXICO: EL CASO DE LA ZARZAMORA

comercializadoras, entre otros. Por lo que, el desempeño de la cadena de suministro de la zarzamora deberá ser alto y todas las empresas que participan en dicha cadena deben ajustarse a la misma para que en el menor tiempo posible se haga llegar el producto a los diversos clientes.

Diversos autores han utilizado o propuesto variables para diversos sectores con la finalidad de medir el desempeño de las cadenas de suministro. A continuación, se muestra un resumen.

\section{Tabla 1}

Resumen de los sectores y las variables de decisión para medir el desempeño de la cadena de suministro

\begin{tabular}{|c|c|c|c|c|c|c|c|c|}
\hline Autor(es) & Sector & $\begin{array}{l}\text { Eficiencia- } \\
\text { costo }\end{array}$ & $\begin{array}{l}\text { Capacidad } \\
\text { de } \\
\text { respuesta } \\
\text { al cliente }\end{array}$ & Flexibilidad & $\begin{array}{l}\text { Calidad } \\
\text { en el } \\
\text { producto }\end{array}$ & $\begin{array}{l}\text { Calidad } \\
\text { en el } \\
\text { proceso }\end{array}$ & $\begin{array}{c}\text { Asignación } \\
\text { y } \\
\text { localización } \\
\text { de la planta }\end{array}$ & $\begin{array}{c}\text { Relaciones } \\
\text { cliente- } \\
\text { proveedor }\end{array}$ \\
\hline Berry y Naim (1996) & Manufacturero & & $\mathrm{X}$ & $\mathrm{X}$ & & & & \\
\hline Murphy et al. (1996) & Manufacturero & $X$ & & & & $\mathrm{X}$ & & \\
\hline Beamon (1998) & Manufacturero & $X$ & $\mathrm{X}$ & $\mathrm{X}$ & $\mathrm{X}$ & & & \\
\hline Beamon (1999) & Manufacturero & $\mathrm{X}$ & $\mathrm{X}$ & $\mathrm{X}$ & $X$ & & & \\
\hline Li y O'Brien (1999) & Manufacturero & $\mathrm{X}$ & $\mathrm{X}$ & $\mathrm{X}$ & $X$ & $\mathrm{X}$ & & \\
\hline Talluri et al. (1999) & Manufacturero & $\mathrm{X}$ & $\mathrm{X}$ & & & $\mathrm{X}$ & & \\
\hline Gunasekaran, et al. (2001) & No especificado & $\mathrm{X}$ & $\mathrm{X}$ & $\mathrm{X}$ & $\mathrm{X}$ & $\mathrm{X}$ & & \\
\hline $\begin{array}{l}\text { Thonemann y Bradley } \\
\text { (2002) }\end{array}$ & Manufacturero & $X$ & X & & & & & \\
\hline Korpela et al. (2002) & No especificado & & & & & & & \\
\hline Lai et al. (2002) & Transportes & $\mathrm{X}$ & $\mathrm{X}$ & $\mathrm{X}$ & $\mathrm{X}$ & & & \\
\hline Talluri y Baker (2002) & Manufacturero & $\mathrm{X}$ & $\mathrm{X}$ & $\mathrm{X}$ & $\mathrm{X}$ & & & \\
\hline Persson y Olhager (2002) & Manufacturero & $\mathrm{X}$ & $\mathrm{X}$ & $\mathrm{X}$ & $\mathrm{X}$ & $\mathrm{X}$ & & \\
\hline Claro et al. (2003) & Hortofrutícola & $X$ & & $\mathrm{X}$ & & & & \\
\hline Wang (2003) & No especificado & $X$ & & & & & & \\
\hline Christy y Grout (1994) & No especificado & $\mathrm{X}$ & $\mathrm{X}$ & & & & & $\mathrm{X}$ \\
\hline Vondouris (1996) & Manufacturero & & & $\mathrm{X}$ & $\mathrm{X}$ & $\mathrm{X}$ & & \\
\hline Van der Vorst (2006) & Alimenticio & $\mathrm{X}$ & $\mathrm{X}$ & $\mathrm{X}$ & $\mathrm{X}$ & $\mathrm{X}$ & & \\
\hline Arana, et al (2010) & Agroalimentario & $\mathrm{X}$ & & & $\mathrm{X}$ & $\mathrm{X}$ & $\mathrm{X}$ & $\mathrm{X}$ \\
\hline Santana y Granillo (2012) & Agroalimentario & $\mathrm{X}$ & $\mathrm{X}$ & $\mathrm{X}$ & X & & & \\
\hline Fattahi y Nookabadi (2013) & Alimenticio & $\mathrm{X}$ & $\mathrm{X}$ & $\mathrm{X}$ & $\mathrm{X}$ & $\mathrm{X}$ & & $\mathrm{X}$ \\
\hline Balfaqih, et al. (2016) & Varios & & $\mathrm{X}$ & $\mathrm{X}$ & $\mathrm{X}$ & $\mathrm{X}$ & & \\
\hline $\begin{array}{l}\text { Voltolini, Pinheiro y } \\
\text { Gouvea (2016) }\end{array}$ & Varios & & $X$ & $X$ & & & & \\
\hline Liu y Liu (2017) & Manufacturero & $\mathrm{X}$ & $\mathrm{X}$ & & & & & $\mathrm{X}$ \\
\hline Equbal y Ohdar (2017) & Varios & $\mathrm{X}$ & & & & $\mathrm{X}$ & & \\
\hline Frecuencia & & 19 & 17 & 15 & 13 & 11 & 1 & 4 \\
\hline
\end{tabular}

Fuente: Elaboración propia. 
FACTORES ASOCIADOS AL DESEMPEÑO DE LA CADENA DE SUMINISTRO AGROALIMENTARIA DE MÉXICO: EL CASO DE LA ZARZAMORA

Derivado del análisis anterior sobre las variables que algunos investigadores expertos en la materia han propuesto y, en algunos casos, aplicado para medir el desempeño de una cadena de suministro, se ha considerado para esta investigación tomar las variables que con mayor frecuencia se han utilizado, sobre todo en los estudios realizados para los sectores de alimentos y agroalimentarios, para conocer su correlación y asociación con respecto al desempeño.

\section{MATERIAL Y MÉTODO}

El objeto de estudio en esta investigación son los productores de zarzamora del Valle de Los Reyes, Michoacán. Esta investigación es cuantitativa de tipo descriptiva-correlacional, descriptiva porque se identifican y describen las variables que están asociadas al desempeño de una cadena de suministro y correlacional porque se establece la relación y asociación entre las variables independientes (calidad en el producto, calidad en el proceso, eficiencia en la utilización de los recursos, flexibilidad a los cambios extraordinarios en el servicio al cliente y la relación clienteproveedor), y la variable dependiente (desempeño de la cadena de suministro de la zarzamora en fresco). Con lo anterior, se podrá demostrar las siguientes hipótesis:

$\mathrm{H}_{0}$ : las variables son independientes.

$\mathrm{H}_{1}$ : las variables no son independientes.

Las hipótesis ayudan a indicar lo que se trata de probar y se definen como explicaciones tentativas del fenómeno investigado y deben formularse a manera de proposiciones (Hernández et al., 2014, p. 104). Cuando el nivel de medida de la característica objeto de contraste no alcanza la escala de intervalo, o cuando no se cumplen los supuestos de los contrastes paramétricos, o cuando lo que interesa es contrastar la distribución de probabilidad, se dispone de una seria de pruebas, genéricamente denominadas no paramétricos, que permiten comprobar las hipótesis planteadas (Moreno y Rodríguez, 2016, p. 282).

El coeficiente de $\rho$ de Spearman plantea las siguientes hipótesis:

$\mathrm{H}_{0}$ : las variables son independientes.

$\mathrm{H}_{1}$ : las variables no son independientes.

Así que, en esta investigación, en primera instancia se realizará la prueba de hipótesis señalada para conocer si existe independencia o no entre las variables independientes y la variable 
FACTORES ASOCIADOS AL DESEMPEÑO DE LA CADENA DE SUMINISTRO AGROALIMENTARIA DE MÉXICO: EL CASO DE LA ZARZAMORA

dependiente, luego se realizará el cálculo del estadístico Gamma de Goodman y Kruskal, el cual indicará el tipo de asociación que existe entre las variables mencionadas.

El coeficiente rho de Spearman se calcula de la siguiente manera:

$$
\rho=1-\frac{6 T}{n\left(n^{2}-1\right)}
$$

Donde:

$$
T=\sum_{i=1}^{n}\left[R\left(x_{i}\right)-R\left(y_{i}\right)\right]^{2}
$$

El coeficiente de correlación oscila entre $-1 \mathrm{y}+1$, el valor de 0 indica que no existe correlación lineal entre las dos variables en estudio (Zimmermann, 2004, p. 344).

El estadístico Gamma de Goodman y Kruskal ( $\mathrm{Y}$ ) es una medida no paramétrica de la fuerza y dirección de asociación que existe entre dos variables medidas en una escala ordinal (Laerd Statistics, 2017). El estadístico gamma ( $\mathrm{Y}$ ) resume la asociación entre dos variables (un predictor y sus respuestas); se encuentra en el intervalo [-1, 1], siendo la diferencia entre las frecuencias muéstrales de pares de observaciones concordantes y discordantes cuando se considera la ordenación tanto del predictor como del resultado. El valor de gamma se puede interpretar como la reducción proporcional en el error cometido al predecir el ordenamiento de los casos en una variable mediante el conocimiento de la ordenación de los casos en otra variable, en lugar de realizar la predicción basándose en una ordenación aleatoria de los casos de las dos variables.

La fórmula para el cálculo de este estadístico es:

$\operatorname{Gamma}(\gamma)=\frac{P-Q}{P+Q}$

Donde:

Y = estadístico gamma de Goodma y Kruskal.

$\mathrm{P}=$ producto de las parejas concordantes.

$\mathrm{Q}=$ producto de las parejas discordantes. 
FACTORES ASOCIADOS AL DESEMPEÑO DE LA CADENA DE SUMINISTRO AGROALIMENTARIA DE MÉXICO: EL CASO DE LA ZARZAMORA

La delimitación espacio-temporal corresponde al Valle de Los Reyes, Michoacán, que abarca los municipios de Los Reyes, Peribán y Tocumbo; y se limita a un punto en el tiempo (2017) puesto que se trabaja con datos de corte transversal. La población objetivo fueron los productores de zarzamora que cuentan con 5 o más hectáreas, siendo un total de 154, a través de la fórmula de poblaciones finitas de acuerdo con Fischer y Espejo (2012, p. 47), se definió una muestra de 48 productores (con un nivel de confianza del 90\% y un error del 10\%), a los cuales se les aplicó un cuestionario de 25 reactivos. Para esta investigación, se utilizó el método probabilístico, a través del muestro aleatorio simple. En esta investigación se hará uso de la escala tipo Likert, la cual en sentido estricto es, una medición ordinal, la cual consiste en un conjunto de ítems presentados en forma de afirmaciones o juicios ante los cuales se pide la reacción de los sujetos (Hernández et al., 2014, p. 238).

En relación a la validez del instrumento aplicado, fue necesario llevar a cabo una prueba piloto, utilizando la validez de expertos.

\section{Tabla 2}

Prueba de confiabilidad por variable y cuestionario completo para la prueba piloto

\begin{tabular}{llc}
\hline No. & \multicolumn{1}{c}{ Variables independientes (VI) y dependiente (VD) } & Alfa de Cronbach $(\alpha)$ \\
\hline 1 & VI-Calidad en el Producto (CPT) & 0.822 \\
2 & VI-Calidad en el Proceso (CPR) & 0.808 \\
3 & VI-Eficiencia en la utilización de los recursos (EFR) & 0.779 \\
4 & VI-Flexibilidad a los cambios extraordinarios en el servicio al cliente & 0.832 \\
& (FLEX) & 0.804 \\
5 & VI-Relación cliente-proveedor (RCP) & 0.855 \\
6 & VD-Desempeño de la cadena de suministro de la zarzamora (DCSZ) & 0.845 \\
\hline
\end{tabular}

Fuente: Elaboración propia con base en investigación de campo y utilizando el software SPSS v19.

Con los resultados obtenidos en la validez y confiabilidad de la prueba piloto, se determina que los valores del Alfa de Cronbach por cada variable independiente y dependiente, así como el cuestionario completo son de aceptables a buenos, según la escala de George y Malley (2003, p. 231). Lo que indica, que el instrumento diseñado puede ser aplicado a los 48 productores de zarzamora. 
FACTORES ASOCIADOS AL DESEMPEÑO DE LA CADENA DE SUMINISTRO AGROALIMENTARIA DE MÉXICO: EL CASO DE LA ZARZAMORA

Para el análisis de datos en esta investigación, se ha utilizado la estadística inferencial, la cual es una parte de la estadística que a través del muestreo infiere conclusiones acerca de la totalidad de una población; asimismo, a través de la prueba de hipótesis se trata de verificar si ciertas suposiciones acerca de la población son ciertas o no (Díaz, 2013, p. 3). Con el software SPSS (Statistical Package for the Social Science) versión 19 se han realizado todos los cálculos: prueba de hipótesis y correlación con el coeficiente rho $\rho$ de Spearman y el grado de asociación con el estadístico gamma y de Goodman y Kruskal.

\section{RESULTADOS}

La aplicación del instrumento de recolección de datos (cuestionario) se realizó durante los meses de mayo a julio del 2017. El 40\% de la muestra se aplicó a través del correo electrónico y resto fue de forma presencial, acudiendo directamente con los productores a sus huertas. Afortunadamente, se logró recabar información de 44 productores, los cuatro restantes no dieron información debido a la confidencialidad.

\section{Tabla 3}

Prueba de confiabilidad por variable y cuestionario completo aplicado a los 44 productores de zarzamora

\begin{tabular}{lcc}
\hline No. & Variables independientes $(\mathrm{VI})$ y dependiente $(\mathrm{VD})$ & Alfa de Cronbach $(\alpha)$ \\
\hline 1 & VI-CPT & 0.849 \\
2 & VI-CPR & 0.794 \\
3 & VI-EFR & 0.810 \\
4 & VI-FLEX & 0.804 \\
5 & VI-RCP & 0.832 \\
6 & VD-DCSZ & 0.837 \\
7 & Cuestionario completo & 0.848 \\
\hline
\end{tabular}

Fuente: Elaboración propia con base en investigación de campo y utilizando el software SPSS v19.

Se aprecia en la tabla anterior que, según la escala establecida por George y Malley (2003, p. 231), las variables independientes y la variable dependiente, así como el cuestionario completo se ubican en un valor de confiabilidad de aceptable a bueno.

Con respecto al nivel educativo, el $42 \%$ cuenta con la educación básica (primaria o y/o secundaria), el 36\% tiene bachillerato y el 20\% licenciatura. El 43\% de los encuestados comentó que tiene de 5 a 7 hectáreas para la producción de zarzamora, el 32\% tiene de 8 a 10 hectáreas 
FACTORES ASOCIADOS AL DESEMPEÑO DE LA CADENA DE SUMINISTRO AGROALIMENTARIA DE MÉXICO: EL CASO DE LA ZARZAMORA

y el resto cuenta con más de 10 hectáreas. El $52 \%$ de los productores tiene de 1 a 5 años de experiencia en la producción de zarzamora, mientras que el 38\% cuenta con una experiencia de 6 a 10 años; el resto tiene una experiencia mayor a 10 años.

El $56.8 \%$ de los productores de zarzamora estuvieron de acuerdo en que la entrega de la mercancía en tiempo y cantidad ayuda a mejorar el desempeño de una cadena de suministro, así como también consideran que sus entregas en tiempo y cantidad de su producto a sus clientes son muy buenas.

El $65.9 \%$ de los encuestados manifestaron estar de acuerdo con que las certificaciones, imagen, presentación, sabor y textura del producto son importantes para un buen desempeño de la cadena de suministro; asimismo se observa que están de acuerdo con que estás características son relevantes para el desempeño de la cadena de suministro.

El 50\% de los productores encuestados señalaron estar de acuerdo en que la trazabilidad y los sistemas de almacenaje, distribución y transporte ayudan a mejorar el desempeño de una cadena de suministro; por lo que, conocer en todo momento la ubicación del producto es de suma importancia para que el desempeño de la cadena de suministro sea el adecuado; debido a que estos elementos son de gran apoyo al desempeño de la cadena.

El $43.2 \%$ de los productores encuestados expuso su acuerdo en que la eficiencia en el uso de los recursos, a través del uso completo de la capacidad instalada es importante para el adecuado desempeño de la cadena.

El $50 \%$ de los productores de zarzamora manifestó estar de acuerdo en que debe haber flexibilidad ante los cambios que el cliente solicita en relación al tiempo y cantidad del producto, para que una cadena de suministro tenga un desempeño adecuado; ante esta situación, consideran que dicha flexibilidad es primordial para que el desempeño de una cadena de suministro sea adecuado.

El 52.3\% de los productores encuestados comentaron que una muy buena relación entre cliente y proveedor es importante para que sea adecuado el desempeño de una cadena de suministro. Lo anterior, indica que la constante comunicación entre clientes y proveedores a través del uso de las Tecnologías de la Información y la Comunicación, ayuda en el adecuado desempeño de una cadena de suministro; por lo que, estuvieron de acuerdo en que el uso de tecnología es 
FACTORES ASOCIADOS AL DESEMPEÑO DE LA CADENA DE SUMINISTRO AGROALIMENTARIA DE MÉXICO: EL CASO DE LA ZARZAMORA

importante para estar en contacto con los clientes y proveedores y que el desempeño de la cadena sea adecuado.

En términos generales, se observó que el 52.3\% de los productores de zarzamora encuestados indicaron que actualmente el desempeño de la cadena de suministro de la zarzamora es bueno; el $38.6 \%$ que es muy bueno y el $9.1 \%$ que es excelente. Lo que hace suponer que, es muy probable que las variables propuestas en esta investigación sean las que se asocian al desempeño de una cadena de suministro agroalimentaria, específicamente del cultivo de la zarzamora.

\section{CONCLUSIONES}

A continuación, se presentan los resultados del coeficiente de correlación de Spearman ( $\rho$ ) y el estadístico de asociación gamma $(\mathrm{\gamma})$ de Goodman y Kruskal.

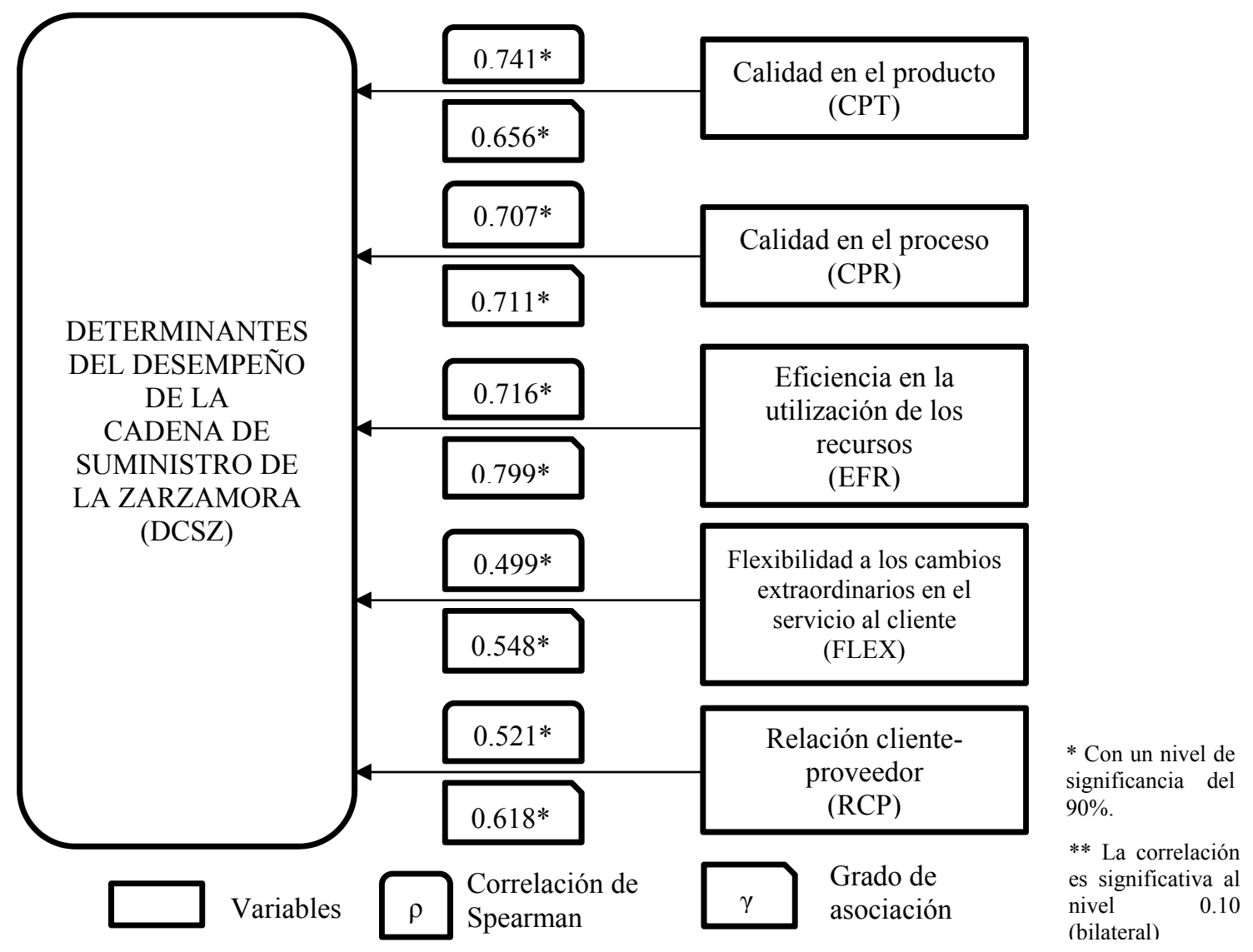

Figura 2. Correlación y grado de asociación de las variables independientes y las variables dependientes de desempeño de la cadena de suministro de la zarzamora.

Fuente: Elaboración propia con base en la investigación de campo utilizando el SPSS v19. 
FACTORES ASOCIADOS AL DESEMPEÑO DE LA CADENA DE SUMINISTRO AGROALIMENTARIA DE MÉXICO: EL CASO DE LA ZARZAMORA

El coeficiente de correlación de Spearman ( $\rho$ ) ayuda a comprobar si las variables independientes CPT, CPR, EFR, FLEX y RCP son independientes a la variable DCSZ o no son independientes. En la figura 3, se muestran los resultados:

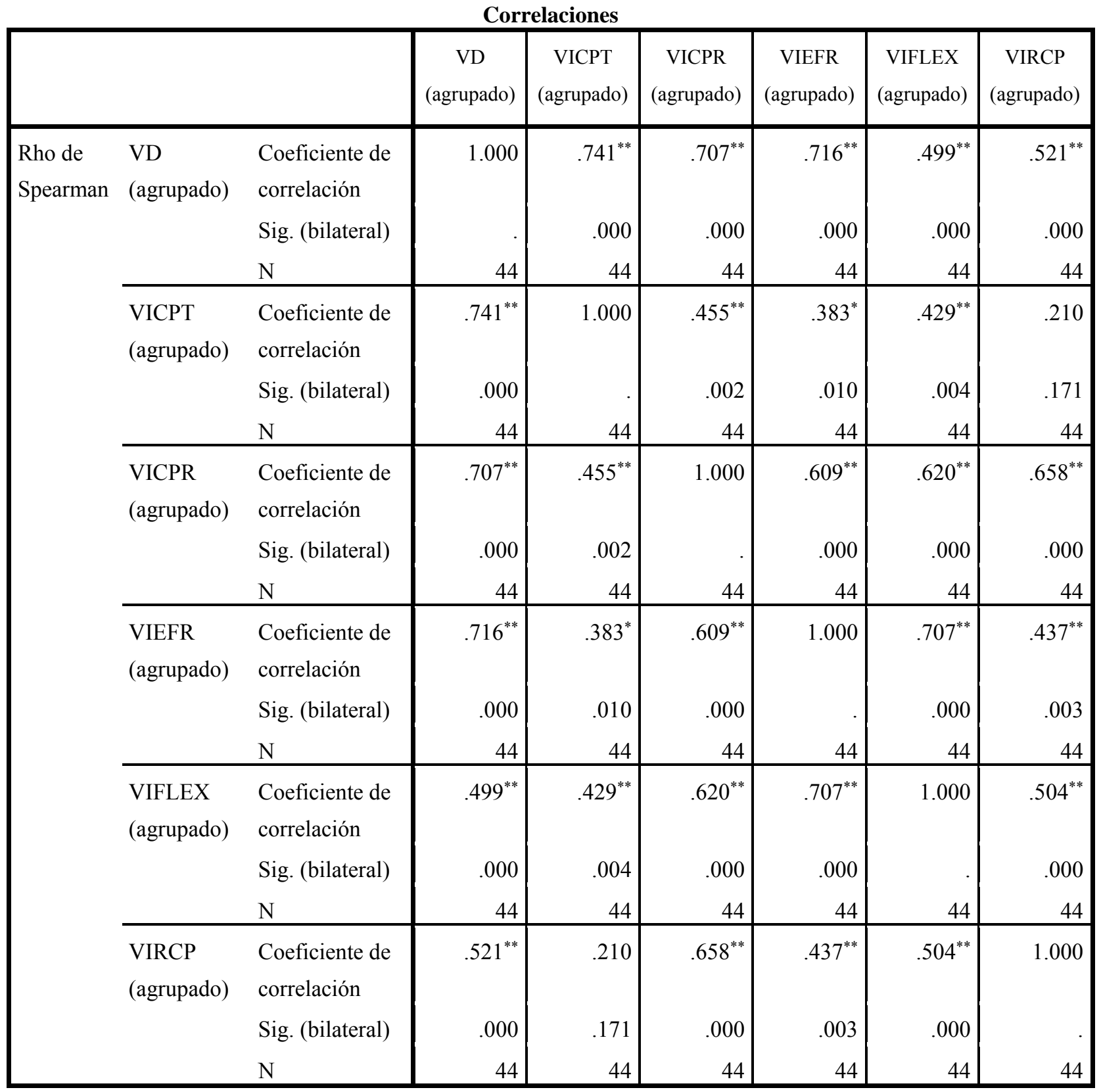

** La correlación es significativa al nivel 0,01 (bilateral).

* La correlación es significativa al nivel 0,05 (bilateral).

Figura 3. Correlaciones entre las variables independientes y las variables dependientes.

Fuente: Elaboración propia con base en la investigación de campo utilizando el SPSS v19. 
FACTORES ASOCIADOS AL DESEMPEÑO DE LA CADENA DE SUMINISTRO AGROALIMENTARIA DE MÉXICO: EL CASO DE LA ZARZAMORA

La hipótesis a probar para el caso de la independencia o no de las variables es:

$\mathrm{H}_{0}$ : las variables CPT, CPR, EFR, FLEX y RCP son independientes a la variable DCSZ.

$\mathrm{H}_{1}$ : las variables CPT, CPR, EFR, FLEX y RCP no son independientes a la variable DCSZ.

Con base en lo que se muestra en la figura 2, se aprecia que se rechaza la hipótesis nula $\left(\mathrm{H}_{0}\right)$, sobre la independencia de las variables; por lo que se acepta la hipótesis alternativa $\left(\mathrm{H}_{1}\right)$, con un nivel de significancia del $90 \%$, lo que indica que las variables no son independientes, por lo que existe una correlación entre ellas.

Derivado del análisis de los resultados, se logró identificar que todas las variables independientes tienen correlación y cierto grado de asociación con la variable dependiente. Con respecto a la relación que cada variable independiente tuvo con la variable dependiente del desempeño de la cadena de suministro de la zarzamora, la variable calidad en el producto (CPT) fue la que tuvo una mayor correlación, seguida por la variable eficiencia en la utilización de los recursos (EFR), luego por la variable calidad en el proceso (CPR), después la variable relación cliente-proveedor (RCP); y por último, la variable flexibilidad a los cambios extraordinarios en el servicios al cliente (FLEX), fue la que menor relación tuvo. Por otro lado, la variable EFR fue la que mayor asociación tuvo con la variable dependiente, seguida por la variable CPR, luego la variable CPT, después la variable RCP, y por último, la variable FLEX.

Con lo anterior, se lograron comprobar la hipótesis de esta investigación, quedando expuesto que las variables no son independientes y que están asociadas al desempeño de la cadena de suministro de la zarzamora en fresco producida en el Valle de Los Reyes, Michoacán.

La teoría establece que las organizaciones deben diseñar métricas e indicadores para medir el desempeño de una cadena de suministro, lo que les permite un control adecuado sobre las variables o factores que mayormente influyen en dicho desempeño. Sin embargo, en relación al sector agroalimentario la situación es un tanto complicada, debido a que el producto principal es un alimento, lo que de manera implícita involucra una serie de factores de especial cuidado como es la vida de anaquel, la seguridad alimentaria, el manejo del producto. Las investigaciones relacionadas con el desempeño de una cadena de suministro agroalimentaria proponen que los factores mayormente relacionados con el desempeño son eficiencia en costos, calidad del alimento (producto) y la flexibilidad en los cambios de entregas (tiempos y cantidades). Esto es para todos los miembros de la cadena de suministro. 
FACTORES ASOCIADOS AL DESEMPEÑO DE LA CADENA DE SUMINISTRO AGROALIMENTARIA DE MÉXICO: EL CASO DE LA ZARZAMORA

De los principales hallazgos de esta investigación se resalta que la literatura relacionada con la medición del desempeño de una cadena de suministro es amplia y que el sector donde predomina este tipo de investigaciones es el manufacturero. Otro hallazgo interesante es, que las variables que más se relacionan con la medición del desempeño son genéricas en diversos sectores, lo que indica que, en su mayoría, existe un acuerdo sobre lo que se debe contemplar en el análisis de una cadena de suministro. Existe una amplia coincidencia sobre las variables asociadas al desempeño, entre los diversos trabajos de investigación en el sector agroalimentario y lo que la evidencia de los productores de zarzamora indica. Es importante resaltar, que los productores de zarzamora están familiarizados con el tema de la cadena de suministro, lo cual revela que tienen un compromiso con su empresa y les interesan los temas que tienen que ver con cuestiones organizacionales en general.

El estado de Michoacán es referente a nivel nacional en la producción de zarzamora en fresco, para el año 2015 representó el 94\% de superficie cosechada a nivel nacional, lo que significó el 94.5\% de la producción en el país. Para el Estado de Michoacán, este cultivo representó el tercer lugar en relación al valor de la producción, sólo por debajo del aguacate y el maíz, primero y segundo lugar, respectivamente para el mismo año. La región de Los Reyes, compuesta por los municipios de Peribán, Los Reyes y Tocumbo, ubicados en el estado de Michoacán, es la zona de mayor producción de la zarzamora en fresco a nivel nacional y estatal. En el año 2015, dicha región tuvo una producción de más del $60 \%$ con respecto a todo el Estado, la tendencia es a la alza, ya que año con año se suman más productores a la siembra, cosecha y comercialización del cultivo de la zarzamora, incluso productores de otros cultivos transitan hacía este cultivo debido a su alta rentabilidad.

En términos generales, las aportaciones por parte de los productores de zarzamora van en la misma dirección a lo que establece y sostiene la teoría; sin embargo, la teoría indica que existen más variables que podrían estar relacionadas y asociadas al desempeño de una cadena de suministro. El estudio y análisis de numerosas variables es interesante e implica un reto mayor, pero la revisión realizada en esta investigación con las variables propuestas brinda un avance bastante significativo, dando lugar a proceder a un estudio continúo a futuro en donde se pueda llevar a cabo la medición del desempeño de la cadena de suministro de la zarzamora tomando en consideración las variables señaladas.

Está investigación permitió identificar que la percepción de los productores sobre el desempeño de la cadena de suministro de la zarzamora es, en términos generales, bueno; sin embargo, es 
FACTORES ASOCIADOS AL DESEMPEÑO DE LA CADENA DE SUMINISTRO AGROALIMENTARIA DE MÉXICO: EL CASO DE LA ZARZAMORA

interesante resaltar que esto no es indicador de que dicho desempeño sea adecuado y que no existan oportunidades de mejora. Los productores de zarzamora expresaron que, en general, entregan en tiempo y cantidad el producto a las empresas comercializadoras de la región, además de que la imagen, presentación, sabor y textura de su producto es buena. En relación a los sistemas de almacenaje, transporte y distribución, la mayoría de los productores tienen dificultades; asimismo, también tienen conflictos para conocer la ubicación de su producto en cualquier momento. La flexibilidad ante cambios en tiempos y cantidades para la entrega del producto es débil por parte de los productores, de igual forma sucede con respecto al manejo de las tecnologías de información en relación con sus clientes.

\section{REFERENCIAS BIBLIOGRÁFICAS}

Agami, N., Mohamed, S. y Mohamed, R, M. (2012). Supply chain performance measurement approaches: review and classification. Journal of Organizational Management Studies, pp. $1-20$.

Arana, J., Jos, B., Onno, O. y Alfons, L. (2010). Relationship characteristics and performance in fresh produce supply chains: the case of the mexican avocado industry. Journal on Chain and Network Science, pp. 1-15.

Balfaqih, H., Zulkifli, N., Nizaroyani, S. y Malak, A. (2016). Review of supply chain performance measurement systems: 1998-2015. Computers in Industry, pp. 135-150.

Beamon, B. (1998). Supply chain design and analysis: models and methods. International Journal of Production Economics, pp. 281-294.

Beamon, B. (1999). Measuring supply chain performance. International Journal of Operations \& Production Management, pp. 275-292.

Berry, D. y Mohamed, N. (1996). Quantifying the relative improvements of redesign strategies in P.C. supply chain. International Journal of Production Economics, pp. 181-196.

Bowersox, D. y David, C. (1996). Logistical management: the integrated supply chain process. Nueva York: McGraw-Hill.

Castañeda, T., Francois, B., Ernesto, S. y Angélica, E. (2009). La concentración de agroindustrias rurales de producción de quesos en el noroeste del Estado de México: un estudio de caracterización. Estudios Sociales (online), 17(34), pp. 73-109.

Claro, D., Geoffrey, H. y Onno, O. (2003). The determinants of relational governance and performance: how to manage business relationhips? Industrail Marketing Management, pp. 703-716. 
FACTORES ASOCIADOS AL DESEMPEÑO DE LA CADENA DE SUMINISTRO AGROALIMENTARIA DE MÉXICO: EL CASO DE LA ZARZAMORA

Council of Logistics Management. (2000). What It's All About. Chicago: Council of Logistics Management.

Chopra, S. y Peter, M. (2001). Supply chain management: strategy, planning and operation. Nueva York: Prentice Hall.

Christopher, M. (1998). Logistics and supply chain management: strategies for reducing costs and improving services. Londres: Pitman Publishing.

Christy, D. y John, G. (1994). Safeguarding supply chain relationships. International Journal of Production Economics, pp. 233-242.

Díaz, A. (2013). Estadística aplicada a la administración y la economía. México: Mc Graw Hill.

Equbal, A. y Ohdar, R. (2017). A comprehensive supply chain performance measurement and evauation (CSCPME) methodology. International Journal of Mechanical and Production Engineering Research and Development, pp. 1-18.

Food and Agriculture Organization of the United Nations. (2009). How to feed the world in 2050. High-Level Expert Forum (pp. 1-35). Roma 12-13, octubre: ONU.

Fattahi, F. y Ali, N. (2013). A model for measuring the performance of the meat supply chain. British Food Journal, pp. 1090-1111.

Fischer, L. y Espejo, J. (2014). Investigación de mercados: un enfoque práctico. México, D.F: OPCOM.

Darren, G. y Paul, Mallery. (2003). SPSS/PC+ step by step: a simple guide and reference. Belmont: Wadsworth Publishing Co.

Gunasekaran, A., Chintan, P. y Tirtiroglu, E. (2001). Performance measures and metrics ina supply chain environment. International Journal of Operations and Production Management, pp. 71-77.

Hernández, R., Fernández, C. y Baptista, P. (2006). Metodología de la Investigación. Ciudad de México: McGraw-Hill Interamericana.

Hidayat, Syarif y Astrellita, S. (2012). Using Supply Chain Operation Reference Model and Failure Mode and Effect Analysis to Measure Delivery Performance of a Distribution System (Case Study: Lotte Mart Indonesia). Jurnal Al-Azhar Indonesia Seri Sains dan Teknologi, pp. 173181.

King, R. y Venturini, L. (2005). Demand for quality drives changes in food supply chains. En Regmi, A., y Mark Gehlhar, New directions in global food markets (págs. 1-81). Washington, DC: USDA.

Jukka, K., Kyläheiko, K., Lehmusvaara, A. y Tuominen, M. (2002). An analytical approach to production capacity allocation and supply chain design. International Journal of Production Economics, pp. 187-195.

Laerd Statistics. (2017). Goodman and Kruskal's gamma using SPSS Statistics. Recuperado de https://statistics.laerd.com/spss-tutorials/goodman-and-kruskals-gamma-using-spssstatistics.php 
FACTORES ASOCIADOS AL DESEMPEÑO DE LA CADENA DE SUMINISTRO AGROALIMENTARIA DE MÉXICO: EL CASO DE LA ZARZAMORA

Kee-hung, L., Ngai, E. y Cheg, E. (2002). Measures for evaluating supply chain performance in transport logistics. Transportation Research, pp. 439-456.

Lambert, D. y Cooper, M. (2000). Issues in supply chain management. Industrial Marketing Management, pp. 65-83.

Le Heron, R. (2003). Creating food futures: reflecting on food governance issues in New Zeeland's agri-food sector. Journal of Rural Studies, pp. 111-125.

Dong, Li., y O’Brien, C. (1999). Integrated decision modelling of supply chain effciency. International Journal of Production Economics, pp. 147-157.

Fuh-hwa, L. y Yu-cheng, L. (2017). A methodology to assess the supply chain performance based on gap-based measures. Computers \& Industrial Engineering, pp. 550-559.

Moreno, E. y Rodríguez, M. (2016). Estimación de parámetros y contraste de hipótesis. Pruebas paramétricos y no paramétricas. En Landero, R., y González, M. Estadística con SPSS y metodología de la investigación (pp. 215-306). Ciudad de México: Trillas.

Murphy, G., Trailer, J. y Hill, R. (1996). Measuring performance in entrepreneurship research. Journal of Business Research, pp. 15.23.

Neely, A., Gregory, M. y Platts, K. (2005). Performance measurement system design. International Jorunal of Operations and Production Management, pp. 1228-1263.

Persson, F. y Olhager, J. (2002). Performance simulation of supply chain design. International Journal of Production Economics, pp. 231-245.

Secretaría de Agricultura, Ganadería, Desarrollo Rural, Pesca y Alimentación. (2010). Retos y oportunidades del sistema agroalimentario de México en los próximos 20 años. DF: SAGARPA.

Santana, F. y Granillo, R. (2012). Identificación de atributos para la medición del desempeño del Sistema Producto Cebada del estado de Hidalgo, México. Científica, 16(1), pp. 11-23.

Servicio de Información Agroalimentaria y Pesquera. (2017). Cierre de la producción agrícola. Recuperado de https://www.gob.mx/siap/acciones-y-programas/produccion-agricola-33119

Talluri, S. y Baker, R. (2002). A multi-phase mathematical programming approach to effective supply chain design. European Journal of Operational Research, pp. 544-558.

Talluri, S., Baker, R. y Sarkis, J. (1999). A framework for designing efficient value chain networks. International Journal of Production Economics, pp. 133-144.

Thenomman, U. y Bradley, J. (2002). The effect of product variety on supply chain perofrmance. European Jorunal of Operational Research, pp. 548-569.

Theodoras, Dimitrios., Lambros, L. y Moschuris, S. (2005). Improving customer service performance within a foos supplier-retailers context. International Journal of Retail and distribution Management, pp. 353-370. 
FACTORES ASOCIADOS AL DESEMPEÑO DE LA CADENA DE SUMINISTRO AGROALIMENTARIA DE MÉXICO: EL CASO DE LA ZARZAMORA

Van der Vorst, J. (2005). Performance Measurement in Agri-Food Supplu Chain Networks: An overview. En Ondersteijn, C., Wijnands, J., Huirne, R., y Van Kooten, O. Quantifying the Agri-Food Supply Chain (p. 242). Dordrecht: Springer.

Van der Vorst, J. (2006). Performance measurement in agrifood supply-chain networks. Logistics and Operations Research Group, pp. 14-24.

Voltolini, A., Pinheiro, E. y Gouvea, S. (2016). Performance measurement for supply chain management: A systematic literature review. 23rd ISPE Inc. International Conference on Transdisciplinary Engineering (pp. 1093-1102). Parana Curitibia: Federal University of Technology.

Voudouris, V. (1996). Mathematical programming techniques to debottleneck the supply chain of fine chemical industries. Computers and Chemical Engineering, pp. 1269-1274.

Wang, N. (2003). Measuring transaction costs: an incomplete survey. Conference on transaction costs (pp. 1-14). Chicago: Ronald Coas Institution.

Zimmermann, F. (2004). Estadística para investigadores. Bogotá: Editorial Escuela colombiana de Ingeniería. 